\title{
Loss of tuberous sclerosis complex 2 sensitizes tumors to nelfinavir -bortezomib therapy to intensify endoplasmic reticulum stress- induced cell death
}

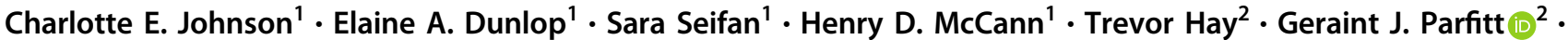 \\ Ashley T. Jones ${ }^{1} \cdot$ Peter J. Giles ${ }^{1} \cdot$ Ming H. Shen ${ }^{1} \cdot J_{\text {Julian R. Sampson }}{ }^{1} \cdot$ Rachel J. Errington $^{1} \cdot$ D. Mark Davies ${ }^{1,3}$. \\ Andrew R. Tee ${ }^{1}$
}

Received: 10 January 2018 / Revised: 10 May 2018 / Accepted: 31 May 2018 / Published online: 6 July 2018

(c) The Author(s) 2018. This article is published with open access

\begin{abstract}
Cancer cells lose homeostatic flexibility because of mutations and dysregulated signaling pathways involved in maintaining homeostasis. Tuberous Sclerosis Complex 1 (TSC1) and TSC2 play a fundamental role in cell homeostasis, where signal transduction through TSC1/TSC2 is often compromised in cancer, leading to aberrant activation of mechanistic target of rapamycin complex 1 (mTORC1). mTORC1 hyperactivation increases the basal level of endoplasmic reticulum (ER) stress via an accumulation of unfolded protein, due to heightened de novo protein translation and repression of autophagy. We exploit this intrinsic vulnerability of tumor cells lacking TSC2, by treating with nelvinavir to further enhance ER stress while inhibiting the proteasome with bortezomib to prevent effective protein removal. We show that TSC2-deficient cells are highly dependent on the proteosomal degradation pathway for survival. Combined treatment with nelfinavir and bortezomib at clinically relevant drug concentrations show synergy in selectively killing TSC2-deficient cells with limited toxicity in control cells. This drug combination inhibited tumor formation in xenograft mouse models and patient-derived cell models of TSC and caused tumor spheroid death in 3D culture. Importantly, 3D culture assays differentiated between the cytostatic effects of the mTORC1 inhibitor, rapamycin, and the cytotoxic effects of the nelfinavir/bortezomib combination. Through RNA sequencing, we determined that nelfinavir and bortezomib tip the balance of ER protein homeostasis of the already ERstressed TSC2-deficient cells in favor of cell death. These findings have clinical relevance in stratified medicine to treat tumors that have compromised signaling through TSC and are inflexible in their capacity to restore ER homeostasis.
\end{abstract}

These authors contributed equally: Charlotte E. Johnson, Elaine A. Dunlop

Electronic supplementary material The online version of this article (https://doi.org/10.1038/s41388-018-0381-2) contains supplementary material, which is available to authorized users.

Andrew R. Tee

teea@cardiff.ac.uk

1 Division of Cancer and Genetics, Cardiff University, Heath Park, Cardiff CF14 4XN, UK

2 European Cancer Stem Cell Research Institute, Cardiff University, Hadyn Ellis Building, Maindy Road, Cardiff CF24 4HQ, UK

3 Department of Oncology, South West Wales Cancer Centre, Singleton Hospital, Swansea SA2 8QA, UK

\section{Introduction}

Cancer cells often display enhanced endoplasmic reticulum (ER) stress, due to inappropriately high levels of protein synthesis, mutational load, and oxidative stress that leads to the accumulation of misfolded protein [1]. The unfolded protein response (UPR) restores ER homeostasis by slowing the rate of protein translation, targeting unfolded protein to degradation pathways (autophagy and the proteasome), and enhancing protein-folding within the ER. If the UPR fails to restore ER homeostasis in a timely manner, cell death will ensue.

Mechanistic target of rapamycin (mTOR) (commonly called mammalian target of rapamycin) functions as a key regulator of protein translation to drive cell growth. Hyperactivation of mTOR complex 1 (mTORC1) elevates ER stress through inappropriately high protein synthesis and an accumulation of unfolded protein [2]. Aberrant mTORC1 activation also potently represses autophagy 
(reviewed in ref. [3]). When autophagy is compromised, the proteasome becomes the primary proteolytic pathway that removes unfolded protein aggregates, thereby restoring ER homeostasis and preventing cell death [4].

Inactivating mutations in either Tuberous Sclerosis Complex 1 (TSC1) or TSC2 give rise to Tuberous Sclerosis (TS), a genetic disorder where patients are predisposed to mTORC1mediated tumors in the brain, kidney, eyes, lung, heart, and skin (for review see ref. [5]). Functional loss of TSC2 and resulting activation of mTORC1 upregulates the proteasome [6]. Consequently, we hypothesize that mTORC1-driven cancers have a higher dependency on the proteasome for survival. A feasible therapeutic strategy might be to inhibit the proteasome with the aim of increasing ER stress beyond a tolerated survival threshold. Supporting this concept, proteasome inhibitors have shown selective cytotoxicity in cancers with heightened mTORC1 [7, 8]. However, the proteasome inhibitor, bortezomib, had little efficacy as a single agent in preventing renal cystadenoma development in a Tsc2+/- mouse model [9]. This study suggested that proteasome inhibition alone is unlikely to cause cytotoxicity in mTORC1-active tumors. We therefore examined the combined effects of bortezomib with nelfinavir. Nelfinavir is an HIV protease inhibitor but has activity against a broad range of cancers. One proposed anticancer action of nelfinavir is through ER stress induction [10]. When combined with nelfinavir, bortezomib has enhanced activity against advanced hematological malignancies such as multiple myeloma, recurrent multiple myeloma, and mantle cell lymphoma [11]. Combined nelfinavir and bortezomib therapy is cytotoxic to breast cancer, acute myeloid leukemia, non-small cell lung cancer, and myeloma cancer models [12-14], prompting several clinical trials (ClinicalTrials.gov: NCT01164709, NCT02188537, NCT01555281).

To stratify therapy, we wanted to determine whether inactivation of TSC2, a key regulator of mTORC1, would sensitize cell and tumor models to combined nelfinavir and bortezomib treatment. Previously we showed that combined treatment with nelfinavir and the autophagy inhibitor, chloroquine, was sufficient to kill cancer cell lines with a high level of mTORC1 [15]. In this study, we reveal that both in vitro and in vivo mTORC1-hyperactive tumor models are sensitive to combined nelfinavir and bortezomib-induced cytotoxicity via ER stress, while normal cells tolerate this drug combination through intact compensatory mechanisms.

\section{Results}

\section{ER stress is elevated upon nelfinavir and bortezomib treatment in Tsc2 $-I-$ MEFs}

To assess ER stress after combined nelfinavir and bortezomib treatment, we analyzed downstream ER stress markers by western blotting. As a control we employed MG132 to inhibit the proteasome. Nelfinavir and bortezomib individually enhanced ER stress in $T s c 2-1-$ mouse embryonic fibroblasts (MEFs), as shown by increased ATF4, CHOP and GADD34 protein, while induction of these proteins in $T s c 2+/+$ MEFs was less evident (Fig. 1a). Combined nelfinavir and bortezomib treatment further elevated the protein levels of ATF4, CHOP, and GADD34 compared to single drug treatment, particularly in the Tsc2-/- MEFs.

We next analyzed Xbpl mRNA splicing, a functional readout of IRE $1 \alpha$ activation and ER stress. Thapsigargin was employed as a control to induce ER stress. We observed more Xbpl mRNA splicing upon nelfinavir treatment in both cell lines. Bortezomib treatment did not cause Xbpl mRNA splicing by itself and did not further enhance splicing when combined with nelfinavir; although nelfinavir- and bortezomib-treated Tsc2-/- MEFs exhibited significantly higher levels of splicing when compared to $T s c 2+/+$ MEFs (Fig. 1b). To confirm this differential ER stress induction between cells with and without $T s c 2$, we examined Chop and Bip mRNA levels (Fig. 1c). In untreated cells, Chop and Bip mRNA in Tsc2-/- MEFs were fivefold and twofold higher, respectively, when compared to $T s c 2+/+$ MEFs, indicating that ER stress is basally elevated in Tsc2-/- MEFs. The Tsc2-/- MEFs were particularly sensitive to nelfinavir treatment (either as a single agent or in combination with bortezomib), resulting in a 2.5-3.3-fold higher level of Chop expression and a 1.8-2.2-fold higher level of Bip expression compared to treatment-matched $T s c 2+/+$ cells. Bortezomib also induced a threefold increase in Chop mRNA and a 2.4-fold increase in Bip mRNA in the Tsc2-/- cells compared to the control cells. These data demonstrate that both nelfinavir and bortezomib treatment induce a higher ER stress burden in Tsc2deficient cells. The PERK inhibitor, GSK2606414, markedly repressed CHOP expression induced by nelfinavir and bortezomib, revealing that these drugs induce an ER stress response through PERK (Fig. 1d).

Given that bortezomib promotes ER stress via proteasomal inhibition and nelfinavir was reported to inhibit the proteasome [16], we examined proteasome activity through either detection of polyubiquitinated protein (Fig. 1a), or levels of chymotrypsin-like activity (Fig. 1e). As expected, bortezomib treatment greatly enhanced the levels of polyubiquitinated protein and effectively reduced chymotrypsin-like activity, indicating robust proteasome inhibition. At $20 \mu \mathrm{M}$, nelfinavir did not show proteasome inhibition in either assay.

Elevation of protein synthesis by mTORC1 hyperactivation likely drives ER stress in Tsc2-deficient cells, but has not been examined to date. We analyzed de novo protein synthesis using pulse-chase $\left[{ }^{35} \mathrm{~S}\right]$-methionine labeling experiments of $T s c 2+/+$ and $T s c 2-/-$ cells in the presence or absence of nelfinavir and bortezomib (Fig. 1f). Tsc2-/- 

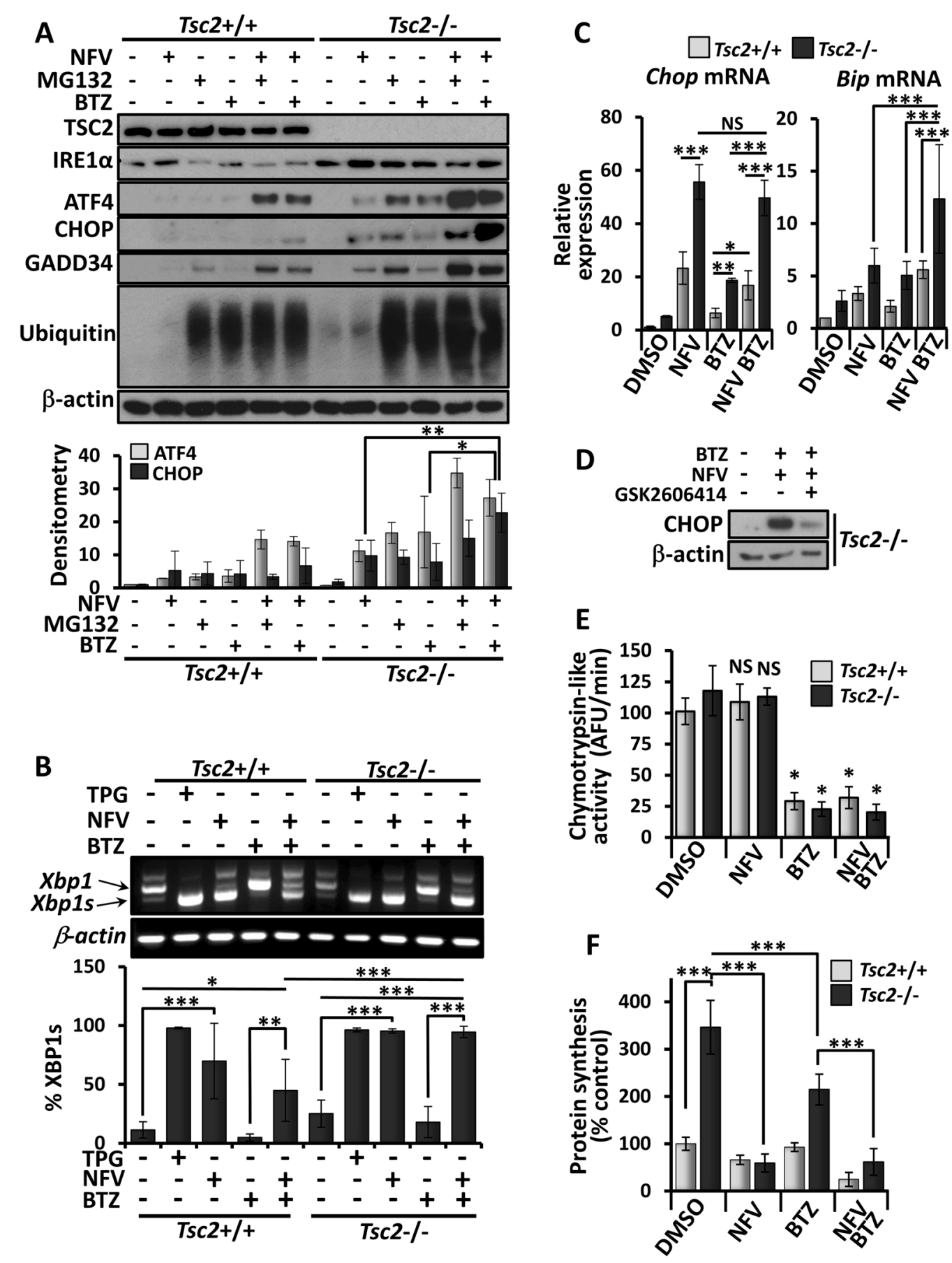

Fig. 1 Nelfinavir markedly enhances ER stress in $T s c 2-/-$ MEFs when combined with proteasome inhibitors. a $T s c 2+I+$ and $T s c 2-I-$ MEFs were treated for $6 \mathrm{~h}$ with either DMSO vehicle, $20 \mu \mathrm{M}$ nelfinavir (NFV), $1 \mu \mathrm{M}$ MG132, $50 \mathrm{nM}$ bortezomib (BTZ), alone or in combination where indicated. Cells were harvested and total protein levels of TSC2, IRE1 $\alpha$, ATF4, CHOP, GADD34, and $\beta$-actin were detected by western blot. Anti-ubiquitin antibodies were used to determine the relative level of polyubiquitinated protein $(n=3)$. b Xbpl mRNA splicing was determined following treatments as indicated. PCR products were resolved on agarose gels (unspliced $=480 \mathrm{bp}$ upper band, spliced $=454 \mathrm{bp}$ lower band, $n=3$ ). The proportion of spliced Xbpl $(X b p 1 s)$ is graphed below. c Chop and Bip mRNA levels were analyzed following $6 \mathrm{~h}$ dual treatment and standardized against Actb mRNA $(n=3)$. d Tsc $2-/-$ MEFs were pretreated with $2 \mu \mathrm{M}$ GSK2606414 (PERK inhibitor) for $30 \mathrm{~min}$, where indicated, before being treated with $20 \mu \mathrm{M}$ nelfinavir and $50 \mathrm{nM}$ bortezomib for $6 \mathrm{~h}$. Protein expression for $\mathrm{CHOP}$ and $\beta$-actin was then determined by western blot analysis $(n=3)$. e The proteasome activity of drug-treated samples, as indicated, was determined by monitoring the turnover of the fluorescent chymotrypsin-like substrate $(n=3)$. Statistics given are relative to the $T s c 2+/+$ DMSO control. f Levels of protein synthesis were determined for control, single and dual treated cells as indicated $(n=5)$ 

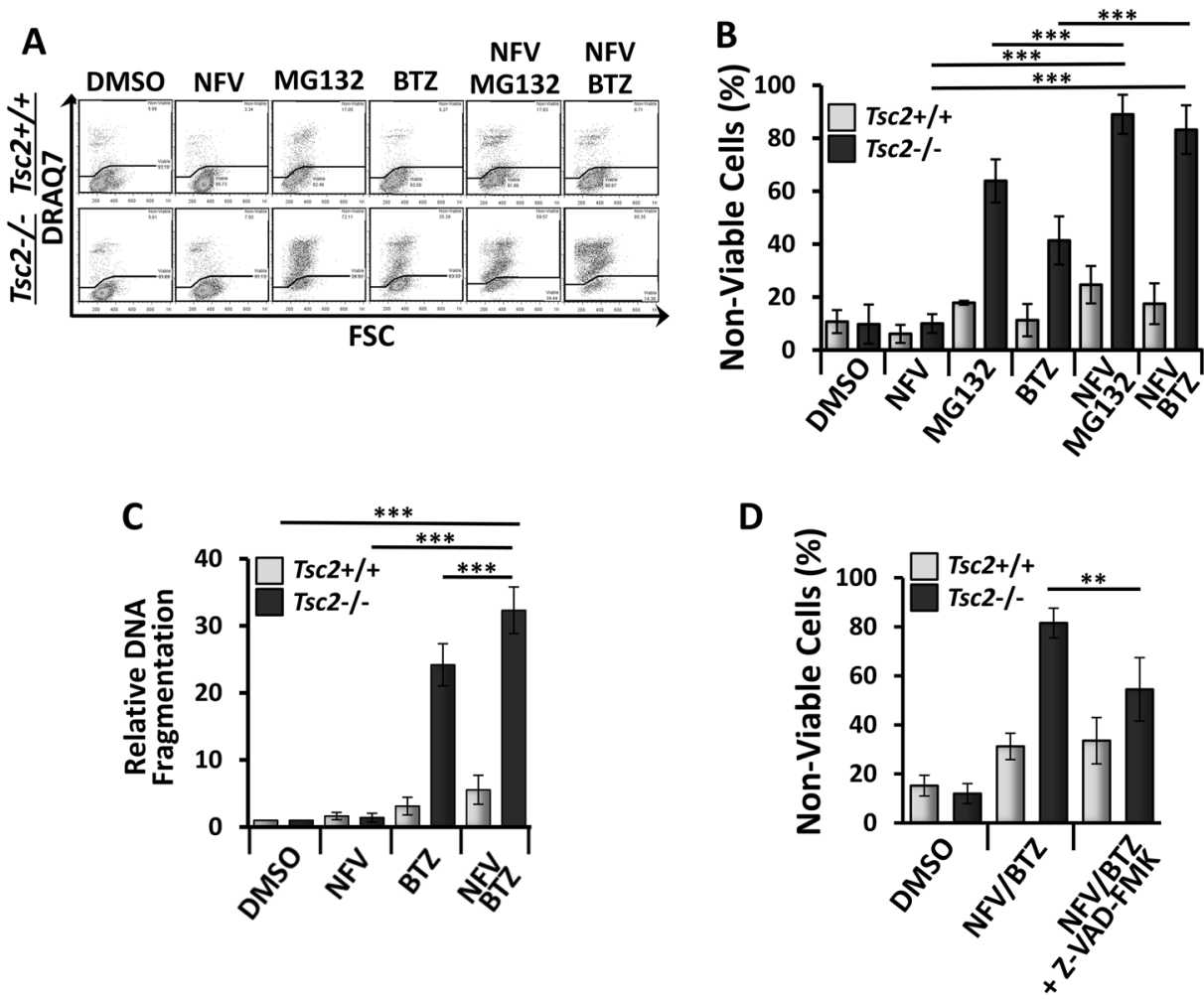

Fig. 2 Nelfinavir enhances the cytotoxicity of bortezomib in $T s c 2-1-$ but not in $T s c 2+/+$ MEFs. a $T s c 2+/+$ and $T s c 2-1-$ MEFs were treated with either DMSO vehicle, $1 \mu \mathrm{M}$ MG132, $50 \mathrm{nM}$ bortezomib (BTZ), $20 \mu \mathrm{M}$ nelfinavir (NFV) alone or in combination where indicated over $24 \mathrm{~h}$. Cells were then subjected to flow cytometry following DRAQ7 staining. DRAQ7 exclusion (below line) represents the viable cell population, while positive DRAQ7 staining (above line) indicates cell death. The number of DRAQ7-stained $T s c 2+1+$ and $T s c 2-1-$ MEFs are graphed in $(\mathbf{b})(n=3)$. $\mathbf{c} T s c 2+/+$ and $T s c 2-/-$ MEFs were

cells had almost fourfold elevation of protein synthesis compared to wild-type, showing that basally ER-stressed $T s c 2-1-$ cells maintain a high level of protein synthesis. After $6 \mathrm{~h}$ of nelfinavir and bortezomib dual treatment, protein translation was markedly reduced.

\section{Proteasome inhibition induced death of Tsc2- deficient cells which is enhanced by nelfinavir}

We speculated that combined nelfinavir and bortezomib treatment might selectively induce cell death in $T s c 2-1-$ MEFs compared to Tsc2 +/+ MEFs. We quantified cell death by flow cytometry with DRAQ7 labeling, which measures cell death via increased membrane permeability (Fig. 2a, b). Both MG132 and bortezomib caused selective cell death in the $T s c 2-/-$ MEFs but not in $T s c 2+/+$ MEFs, revealing that cells devoid of $T s c 2$ are dependent on the proteasome for their survival. Combined nelfinavir and bortezomib treatment enhanced cell death in the Tsc2-1MEFs $(83.2 \% \pm 9.2$ cell death), with minimal toxicity in the $T s c 2+/+$ MEFs $(17.5 \% \pm 7.7)$. The low level of cell death treated for $24 \mathrm{~h}$ with either DMSO, $20 \mu \mathrm{M}$ nelfinavir (NFV), $50 \mathrm{nM}$ bortezomib (BTZ) as single agents or in combination, as indicated, and then subjected to DNA fragmentation assays $(n=5)$. d $T s c 2+/+$ and $T s c 2-1-$ MEFs were treated for $24 \mathrm{~h}$ with $20 \mu \mathrm{M}$ NFV combined with $50 \mathrm{nM} \mathrm{BTZ}$ in the presence or absence of $20 \mu \mathrm{M}$ Z-VAD-FMK and analyzed for cell death by flow cytometry with DRAQ7 staining. The number of DRAQ7-stained $T s c 2+1+$ and $T s c 2-1-$ MEFs are graphed $(n=3)$

in the $T s c 2+/+$ MEFs is not significantly different to the DMSO control. A similar pattern was observed for the nelfinavir/MG132 combination. To validate these findings, we utilized $T s c 2$-deficient and re-expressing ELT3 rat smooth muscle cells [17]. These results mirrored that seen in the Tsc2-/- MEFs (Supplementary Figure 1A and 1B).

To further examine cell death we quantified DNA fragmentation (Fig. 2c). We observed significant DNA fragmentation in Tsc2-I- MEFs after bortezomib treatment that was further enhanced when combined with nelfinavir. No DNA fragmentation was evident with nelfinavir treatment alone. Additionally, we observed cleavage of caspase 8, caspase 3, and PARP in Tsc2-1- MEFs upon treatment with bortezomib alone or cotreatment with nelfinavir and proteasome inhibitors, while no marked cleavage was seen in wild-type cells (Supplementary Figure 1C). We could partially but significantly rescue $T s c 2-/-$ MEFs from nelfinavir/bortezomib-induced cell death by inhibiting apoptosis with the pan caspase inhibitor, Z-VAD-FMK, suggesting cell death is partly mediated through caspase activation (Fig. 2d). 

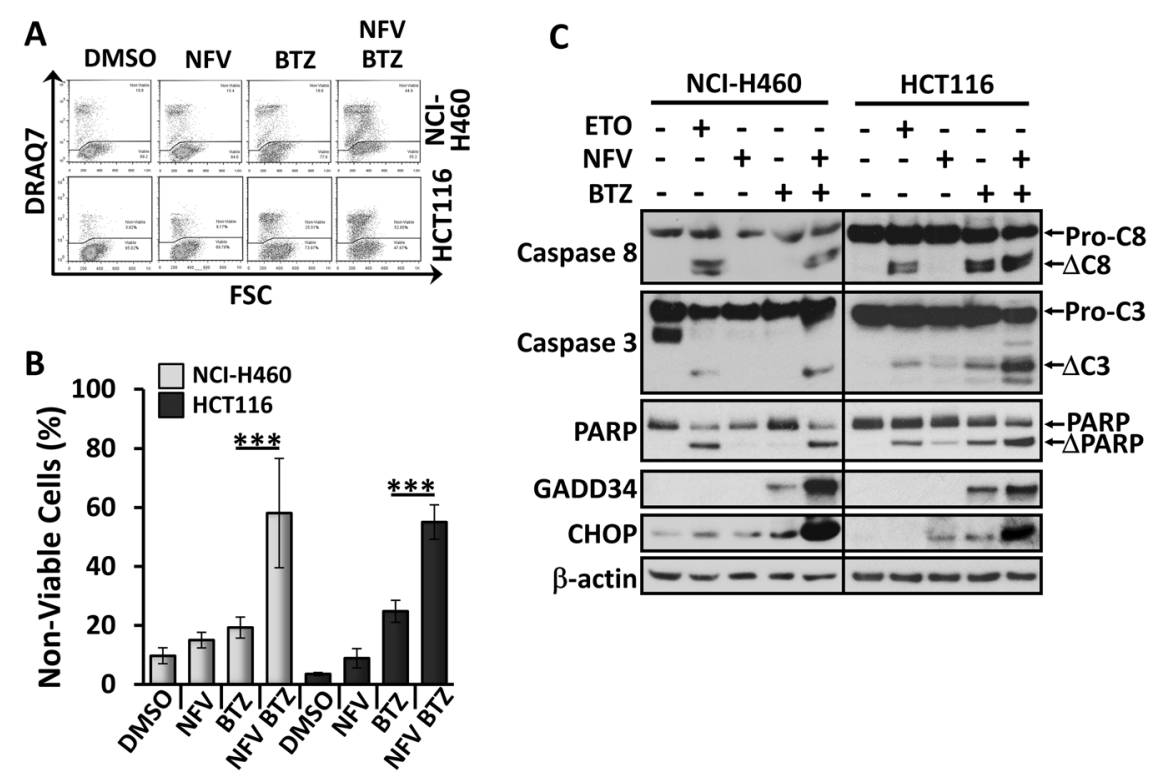

Fig. 3 Nelfinavir enhances the cytotoxicity of bortezomib in human lung and colon cancer cell lines. a NCI-H460 lung cancer and HCT116 colon cancer cells were treated with either DMSO vehicle, $50 \mathrm{nM}$ bortezomib (BTZ), $20 \mu \mathrm{M}$ nelfinavir (NFV) alone, or NFV combined with BTZ over $24 \mathrm{~h}$. Cells were then subjected to flow cytometry with DRAQ7 staining. DRAQ7 exclusion (below line) represents the viable

To determine whether other sporadic cancer cell lines were sensitive to combined nelfinavir and bortezomib treatment, we examined human NCI-H460 lung cancer and HCT116 colon cancer cell lines, which both have elevated levels of mTORC1 signaling. Both cell lines showed sensitivity to the treatment (Fig. 3a, b). Combined treatment caused cell death at levels of $58.1 \% \pm 18.5$ in NCI-H460 cells and $55.1 \% \pm 5.8$ in HCT116 cells, significantly higher than with either agent alone. Both cell lines showed a higher level of caspase 8, caspase 3, and PARP cleavage following dual treatment when compared to single drug treatments (Fig. 3c). Elevated levels of CHOP and GADD34 were observed in nelfinavir- and bortezomib-treated cells, suggesting that cell death was likely mediated through the ER stress pathway.

\section{Synergy of nelfinavir and bortezomib in inducing cell death in Tsc2 $-I-$ MEFs}

We next assessed synergy between nelfinavir and bortezomib to induce cell death. Tsc2+/+ and Tsc2-I- MEFs were treated with nelfinavir and bortezomib at a range of concentrations, both individually and in combination. Cells were analyzed by flow cytometry using DRAQ7 (Fig. 4a-d) revealing that nelfinavir has little cytotoxicity as a single agent, especially at low doses (Fig. 4a), while bortezomib potently induces cell death, more so in $T s c 2-/-$ than $T s c 2$ $+/+$ cells (Fig. 4b). While Tsc $2+/+$ MEFs tolerated high concentrations of nelfinivir and bortezomib in combination (Fig. 4c), Tsc2-/- MEFs were acutely sensitive to lower

cell population, while positive DRAQ7 staining (above line) indicates cell death. The number of DRAQ7-stained cells are graphed in (b) (n $=3)$. $\mathbf{c}$ With the addition of Etoposide $(100 \mu \mathrm{M})$, cells were treated as in (a) and total protein levels of Caspase 8 (CASP8), Caspase 3 (CASP3), PARP, GADD34, CHOP and $\beta$-actin were measured by western blot analysis $(n=3)$

concentrations (Fig. 4d). CompuSyn software was used to calculate combination index (CI) values based on mean cell death, which are shown as Chou-Talalay plots for $T s c 2+/+$ (Fig. 4e) and Tsc2-/- (Fig. 4f) MEFs. Values below $\mathrm{CI}=$ 1 indicate synergy, showing that nelfinavir and bortezomib act synergistically to induce cell death in $T s c 2-/-$ MEFs at all concentrations used in this experiment.

\section{Nelfinavir and bortezomib inhibit tumor spheroid formation and outgrowth of Tsc2-I- cells}

Based on the concentrations that demonstrated synergy in Tsc2-/- MEFs, we utilized $20 \mathrm{nM}$ bortezomib with $20 \mu \mathrm{M}$ nelfinavir in tumor formation assays. Nelfinavir alone did not impact colony formation and growth, but bortezomib treatment impaired growth by $36 \pm 12 \%$ (Fig. $5 \mathrm{a}$ ). When bortezomib was combined with nelfinavir, tumor growth was completely inhibited. To investigate whether nelfinavir and bortezomib could kill established tumors, Tsc2-/- MEFs were cultured as spheroids using 3D cell culture before being treated over $96 \mathrm{~h}$. Nelfinavir and bortezomib as single drug treatments and in combination were compared to the mTORC1 inhibitor, rapamycin. Due to the prolonged time of treatment compared to $2 \mathrm{D}$ experiments, we used lower concentrations of nelfinavir $(10 \mu \mathrm{M})$ and bortezomib $(10 \mathrm{nM})$. Cell death was measured by DRAQ7 staining (Fig. 5b) and compared to spheroid size (Fig. 5c). Combined nelfinavir and bortezomib treatment caused a significant increase in DRAQ7 staining compared to both the single drug treatments 

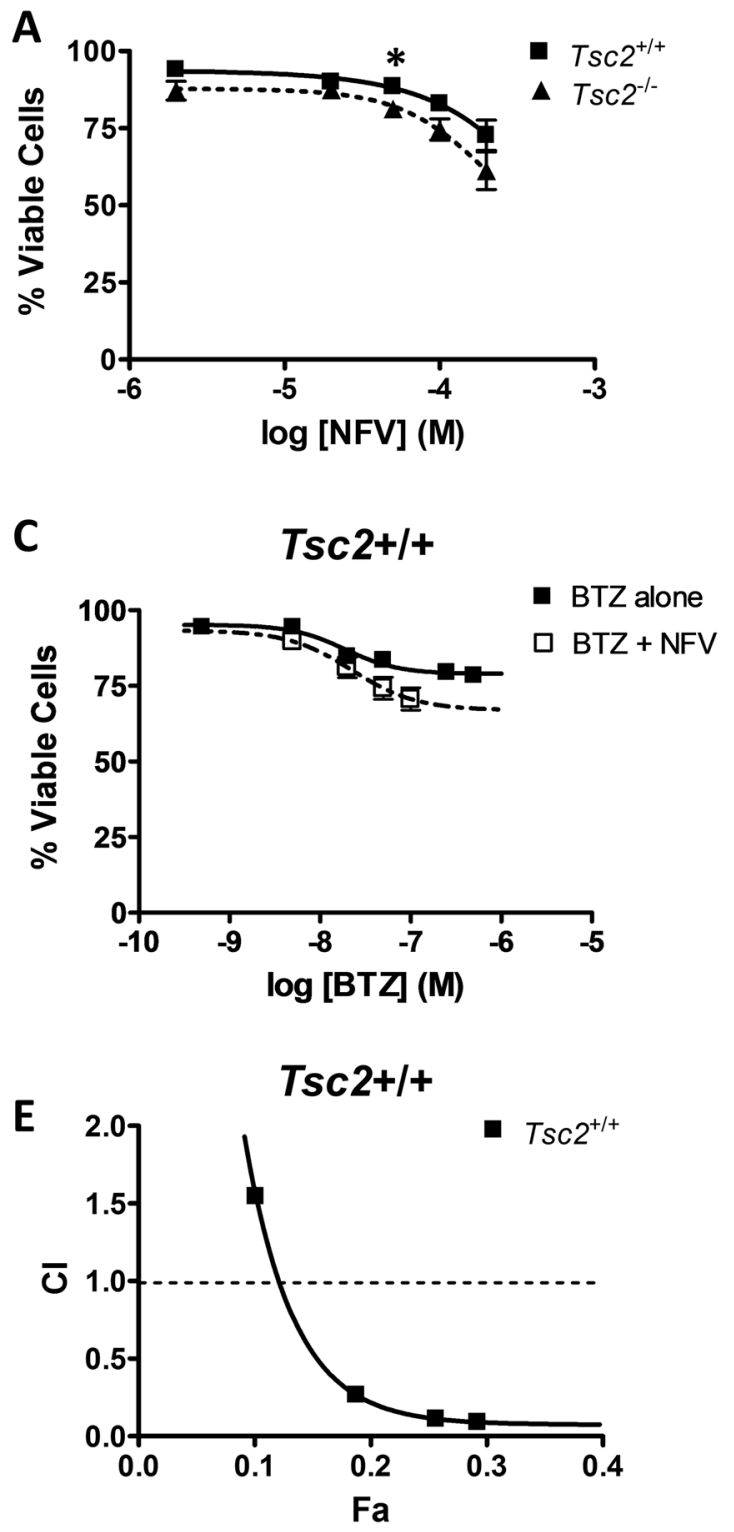

Fig. 4 Nelfinavir and bortezomib synergize to selectively kill $T s c 2-/-$ MEFs. Dose response curves were performed in $T s c 2+1+$ and $T s c 2$ $-1-$ MEFs using flow cytometry as a readout of cell death for nelfinavir (a), bortezomib (b) and the combination $(\mathbf{c}, \mathbf{d})(n=3)$. Synergy

and rapamycin. Rapamycin visibly shrank overall spheroid size, whereas treatments with either nelfinavir or bortezomib did not. To further determine viability, treated spheroids shown in Fig. 5b were plated into 2D cell culture systems and allowed to regrow without the presence of drug. Spheroid outgrowth was measured over $72 \mathrm{~h}$ (Fig. 5d, graphed in 5E). Rapamycin-treated spheroids, although shrunken, still contained viable cells that rapidly grew out into culture. Spheroids treated with either nelfinavir or bortezomib also grew back, while there was no evidence of outgrowth in the combined nelfinavir and bortezomib treatment. The lack of cell recovery and the high level of DRAQ7 staining indicate that combined treatment with nelfinavir and bortezomib

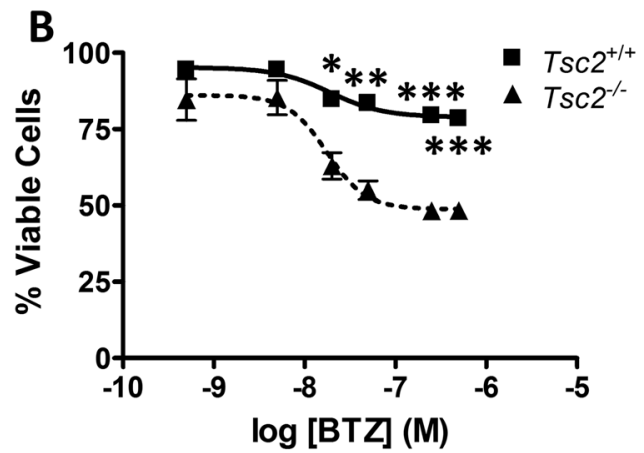

D Tsc2-/-

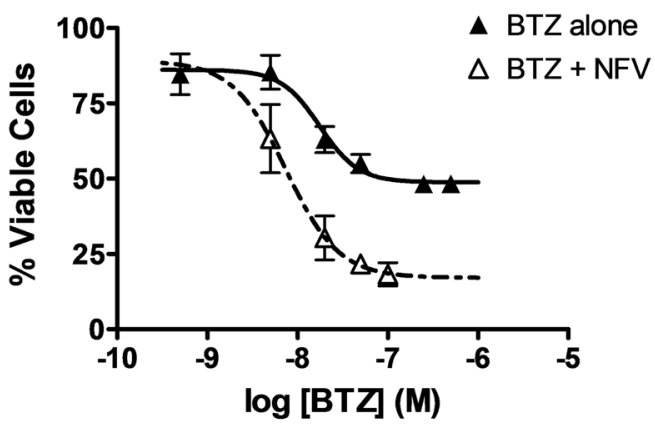

Tsc2-/-

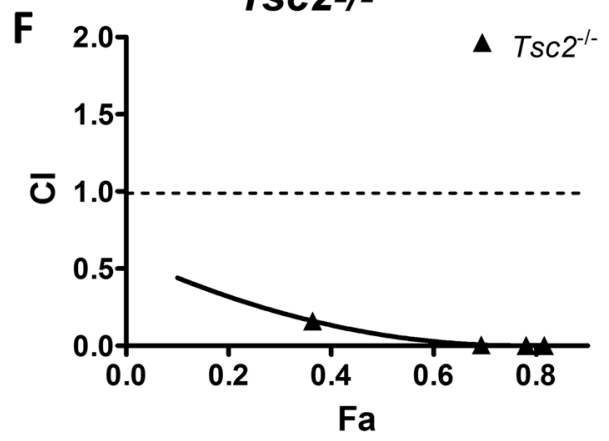

was assessed by examining cell death across a range of bortezomib concentrations, with or without $20 \mu \mathrm{M}$ nelfinavir and calculated using CompuSyn software $(\mathbf{e}, \mathbf{f})$. Graphs show mean \pm S.E.M.

effectively prevents re-growth of spheroids through induction of cell death, whereas rapamycin shrank spheroids that then regrew when treatment was withdrawn, as previously reported in clinical studies with rapalogues $[18,19]$. The effect of nelfinavir and bortezomib in $T s c 2-/-$ MEFs was validated in ELT3-V3 cells that showed a similar response (Supplementary Figure S2A-C). To further investigate how drugs affected tumor size and integrity, phalloidin (green) was used to stain the actin cytoskeleton, and DRAQ7 (far red) to counterstain nuclei, following drug treatment of Tsc2-/- MEFs. Figure $5 \mathrm{f}$ shows that rapamycin-treated spheroids retain a similar degree of actin fluorescence as DMSO-treated controls, whereas nelfinavir and bortezomib-treated spheroids exhibit 
A

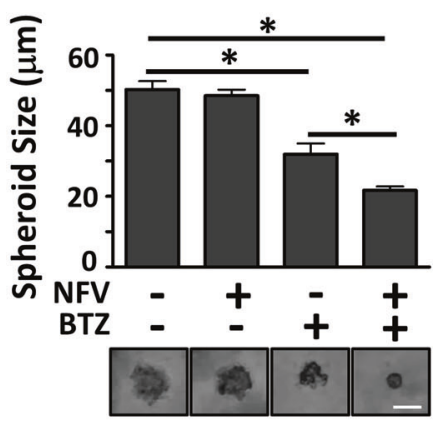

B
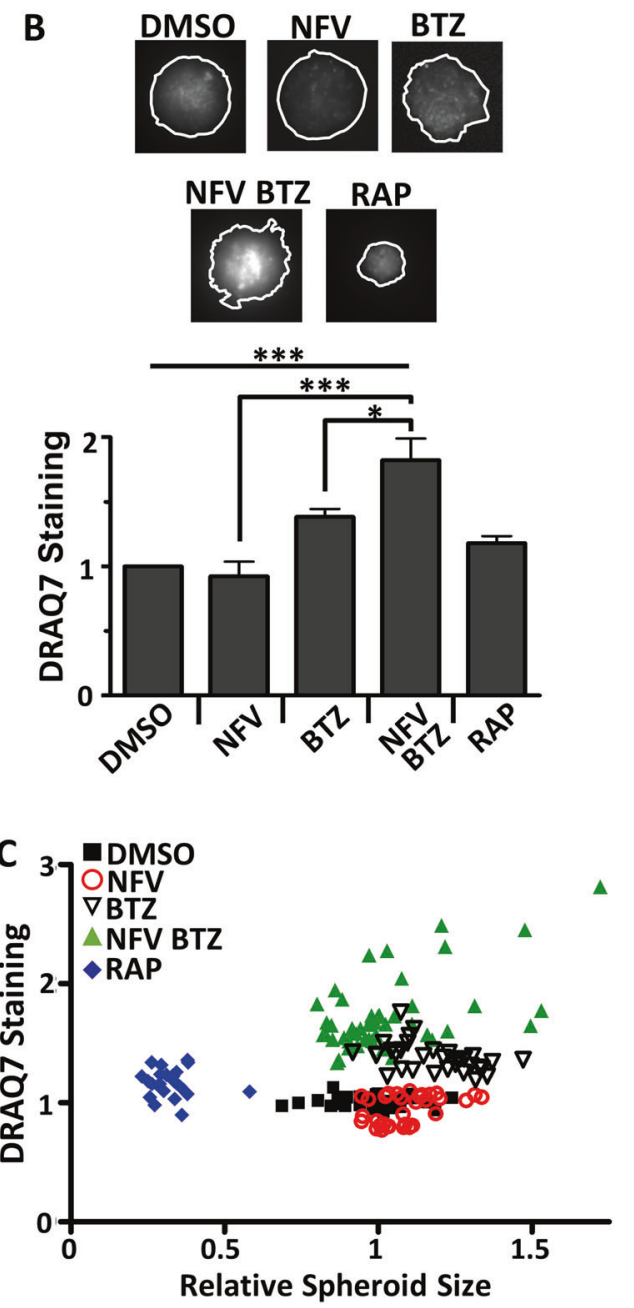

Fig. 5 Nelfinavir and bortezomib prevent tumor spheroid growth in $T s c 2-/-$ MEFs. a Tsc2-/- MEFs were plated in soft agar and treated over 11 days with either $20 \mu \mathrm{M}$ nelfinavir (NFV), $20 \mathrm{nM}$ bortezomib (BTZ), as single agents or in combination. Images of the colonies were taken and the diameters measured using ImageJ. Scale bar is $20 \mu \mathrm{m}(n$ $=4$, with $>100$ spheroids measured per condition, per replicate). $\mathbf{b}$ Tsc2-/- MEF spheroids were treated with DMSO vehicle control, $10 \mu \mathrm{M}$ nelfinavir combined with $10 \mathrm{nM}$ bortezomib (NFV BTZ), or $25 \mathrm{nM}$ rapamycin (RAP), for $96 \mathrm{~h}$. DRAQ7 was added for the final $36 \mathrm{~h}$ to monitor cell death before images were taken and quantified ( $n \geq 3$, average of 12 spheroids per condition per replicate). c Spheroid diameter was determined from phase contrast images of (b) after $96 \mathrm{~h}$
D
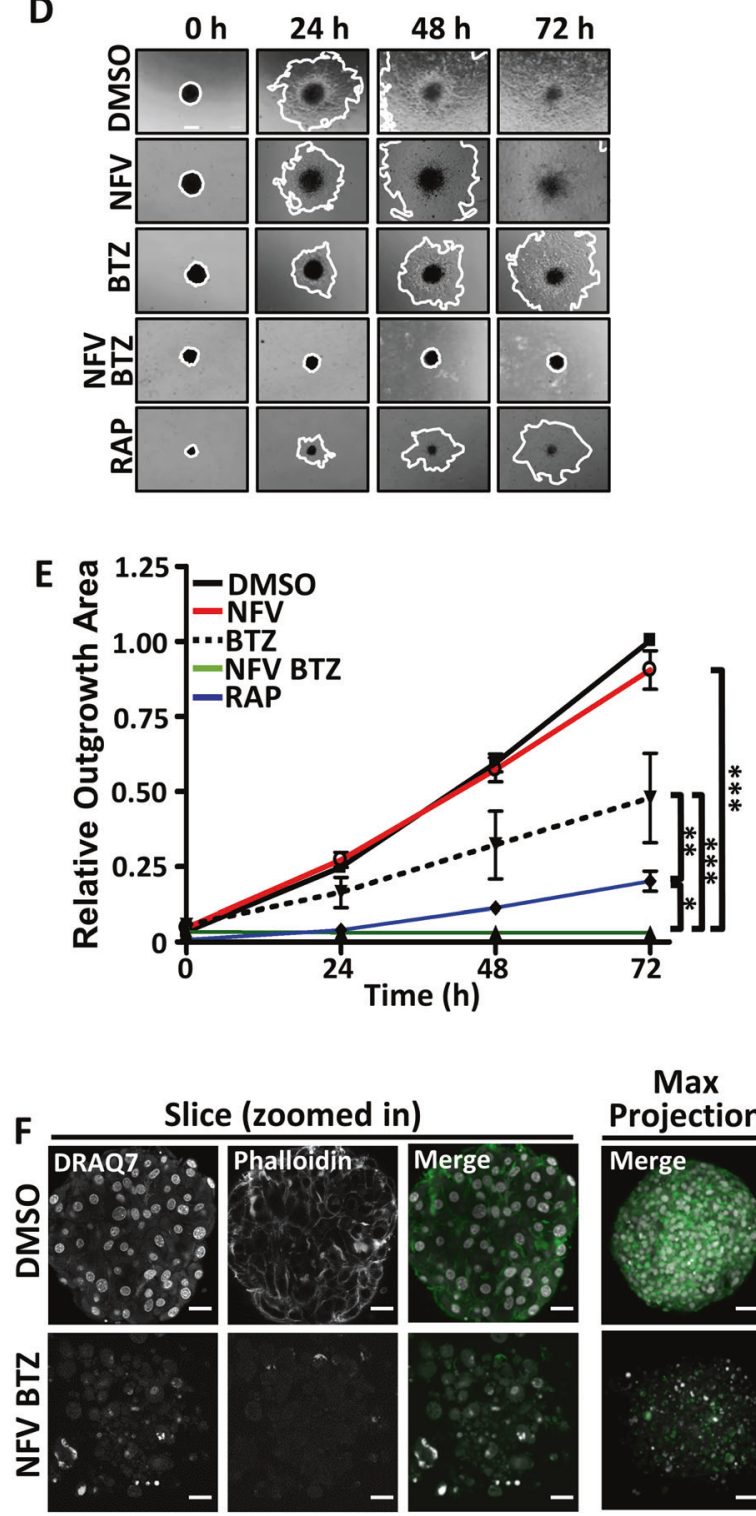

Slice (zoomed in)
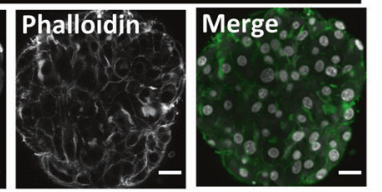

Max

Projection
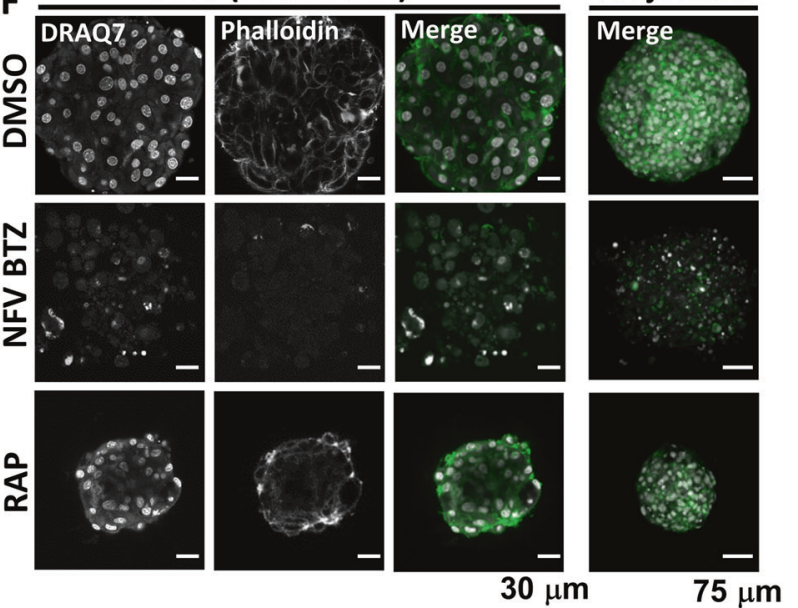

drug treatment and plotted against DRAQ7 staining intensity. d Spheroids were re-plated onto standard tissue culture plates and grown under drug-free conditions. Images were taken every $24 \mathrm{~h}$ and the area of outgrowth calculated using ImageJ. Scale bar is $200 \mu \mathrm{m}$. Relative outgrowth areas are graphed in (e). Statistics compare the $72 \mathrm{~h}$ timepoint. Graphs in (a), (b), and (e) shown mean \pm S.E.M. f Treated spheroids were stained using phalloidin (actin-green in merged images) and DRAQ7 (nuclei-white) and imaged using confocal microscopy. A representative slice $(\times 63$ oil lens) through the spheroid is shown with a scale bar of $30 \mu \mathrm{m}$, alongside the maximum projection ( $\times 20$ lens) with a scale bar of $75 \mu \mathrm{m}$ 
comparatively less actin and nuclear staining. The weak nuclear staining and the collapse of the nuclear envelope is indicative of DNA fragmentation and suggests that nelfinavir -bortezomib is killing cells rather than causing senescence. These data support our findings showing that rapamycin shrinks tumors, but without cytotoxic effects, while nelfinavir -bortezomib treatment is effective at causing cell death.

\section{Nelfinavir and bortezomib downregulates prosurvival and upregulates proapoptosis genes, likely mediated through ER stress}

To better understand the early changes that nelfinavir and bortezomib cause to gene expression, RNA sequencing was performed in $T s c 2+/+$ and $T s c 2-/-$ MEFs after $6 \mathrm{~h}$ of combined treatment or DMSO control. Figure 6a shows genes associated with ER stress, a selection of which is highlighted graphically in Fig. 6b (raw data in Supplementary Table 1). Tsc2-1- MEFs expressed higher basal levels of ER stress genes shown in the panel (Fig. 6a, Supplementary Figure S3A and Supplementary Table 2). Following nelfinavir and bortezomib treatment, expression was further increased (Supplementary Figure S3B and Supplementary Table 3), with expression in $T s c 2-1-$ cells mostly significantly higher than that of the $T s c 2+/+$ MEFs (Fig. 6b). Figure 6c describes the changes of prosurvival and prodeath genes in $T s c 2+/+$ vs. Tsc2-1- dual-treated MEFs (genes selected based on AmiGO "cell death"). Figure 6c shows expression of prosurvival genes to be decreased, and prodeath genes to be increased in drug-treated $T s c 2-1-$ cells compared to treated $T s c 2+/+$ MEFs. The overall RNA sequencing data are shown in a volcano plot (Fig. 6d and Supplementary Table 4), with the genes in Fig. 6c highlighted. To validate that $T s c 2+/+$ MEFs could efficiently restore ER homeostasis, while $T_{s c 2}$ -/- MEFs could not, we carried out a time course of nelfinavir-bortezomib treatment (Fig. 6e). We observed a strong increase in ATF4 and CHOP protein in both cell lines at $6 \mathrm{~h}$ of treatment, which was downregulated by $16 \mathrm{~h}$ to a level that was not significantly different to untreated. However, after $24 \mathrm{~h}$ of treatment, the protein expression of ATF4 and CHOP was enhanced in the $T s c 2-/-$ MEFs, suggesting an inability to efficiently restore ER homeostasis. In contrast, the protein levels of ATF4 and CHOP remained low in the $T s c 2+/+$ MEFs after $24 \mathrm{~h}$.

\section{Nelfinavir and bortezomib reduced tumor volume in ELT-V3 mouse xenografts, correlating with increased CHOP expression}

To determine the antitumor efficacy of nelfinavir and bortezomib in vivo, mice bearing Tsc2-null ELT3 xenograft tumors were treated with the drugs as single agents or in combination. Seventeen days after commencement of treatment, combined nelfinavir and bortezomib decreased tumor growth by approximately $70 \%$ which was a significant decrease compared with vehicle-treated mice (Fig. 7a). The single agent treatments slowed tumor growth but not significantly. While combined treatment of nelfinavir and bortezomib is well tolerated in patients [11], it was not well tolerated in mice. In the combination group, 11/14 mice died or were euthanized due to excessive toxicity compared with $2 / 14$ in the vehicle-treated group, $5 / 14$ in the nelfinavir-alone group and 5/14 in the bortezomib-alone group. Immunohistochemical analysis of xenograft tumor tissue sections revealed a modest increase in CHOP-positive cells after nelfinavir and bortezomib combined treatment (Fig. 7b). The heterogeneity of CHOP staining likely reflects cycles of ER stress induction and recovery in these cells. By western blot analysis, a higher level of ATF4 protein and PARP cleavage was observed in tumors from mice that were treated with both nelfinavir and bortezomib (Fig. 7c), indicating an elevated level of ER stress and cell death upon combined treatment.

\section{Discussion}

This study uses clinically relevant drugs that could be repositioned to treat tumors displaying high ER stress and mTORC1. We reveal that mTORC1-overactive cells are sensitive to nelfinavir and bortezomib treatment. Nelfinavir and bortezomib act to amplify ER stress and synergize to promote cell death. While wild-type cells tolerate this drug combination with minimal cell death, cytotoxicity in $T s c 2$-deficient cells is evident at low drug concentrations and is likely attributable to their inability to manage ER stress. Indeed, we see that ER stress is not fully restored in the $T s c 2$-deficient cells after $24 \mathrm{~h}$ of combined drug treatment, as observed by a reoccurrence of ATF4 and CHOP protein expression (Fig. 6e). Tsc2-deficient cells were reported to have a truncated ER stress response [20], which fits with our observation that cells lacking functional $T s c 2$ are compromised in their ability to restore ER homeostasis. TSC2 functions as an important component of the survival arm during ER stress as it is positioned downstream of several ER stress-mediated survival pathways. One pathway involves GADD34, which recruits protein phosphatase 1 (PP1) to TSC2, dephosphorylating and activating TSC2 to repress mTORC1. We observed high protein levels of GADD34 after ER stress induction in all our cell lines, more so in $T s c 2-1-$ MEFs.

Normally, protein synthesis is downregulated upon ER stress as a strategy to prevent further build-up of unfolded protein within the ER. We observed that $T s c 2$-deficient cells have elevated protein synthesis despite higher background levels of ER stress, with a 3-4-fold increase in protein synthesis in Tsc2-/- MEFs compared to wild-type (Fig. 


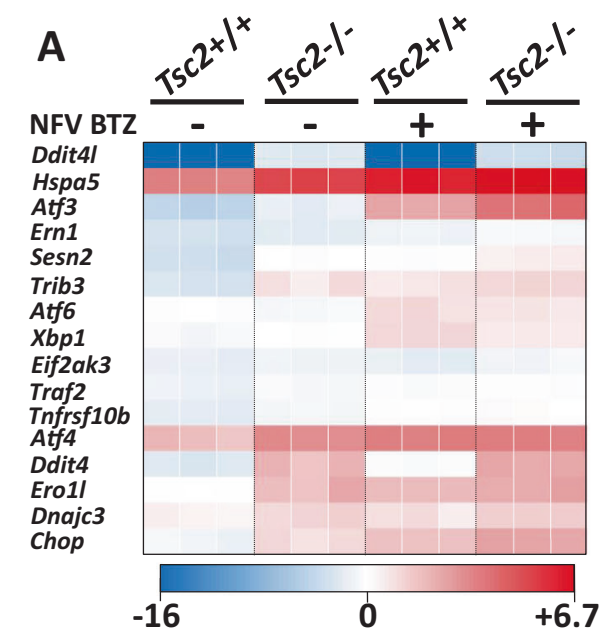

B $\quad$ DTsc2+/
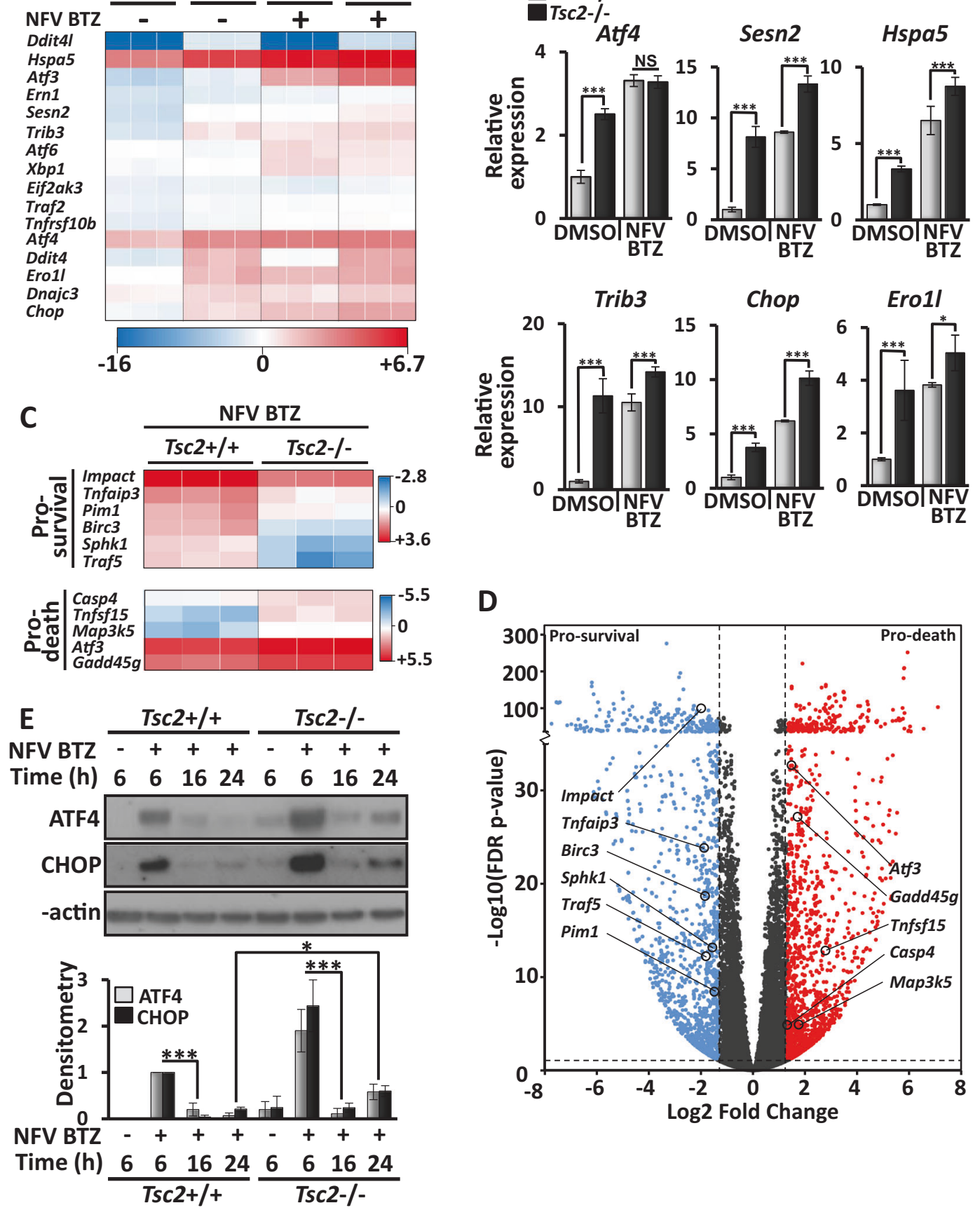

Fig. 6 Nelfinavir and bortezomib upregulate early response genes for ER stress and cell death in Tsc2-I- MEFs, while prosurvival genes are downregulated. $T s c 2+/+$ and $T s c 2-I-$ MEFs were treated with either DMSO vehicle or combined nelfinavir $(20 \mu \mathrm{M})$ and bortezomib $(50 \mathrm{nM})$ for $6 \mathrm{~h}$ before processing for RNA sequencing $(n=3)$. a Heatmap of a panel of ER stress-linked genes, a selection of which are highlighted graphically in (b). c Paired heatmaps from dual treated

1f). Elevated levels of protein synthesis likely enhances ER stress within the $T s c 2$-deficient cells. As well as promoting translation, mTORC1 hyperactivation increases the activity of the proteasome while inhibiting autophagy [6].

cells showing early response genes linked to cell survival and death which are highlighted in a volcano plot (d). e $T s c 2+/+$ and $T s c 2-/-$ MEFs were treated with either DMSO vehicle or combined nelfinavir $(20 \mu \mathrm{M})$ and bortezomib $(50 \mathrm{nM})$ for 6,16 , and $24 \mathrm{~h}$ before extracting protein for western blot and probing for ATF4, CHOP, or $\beta$-actin $(n=3)$

Downregulation of autophagy means that the proteasome becomes the principal mechanism to reduce ER stress via protein degradation in cells lacking $T s c 2$. However, the proteasome inhibitor, bortezomib, had a lack of in vivo 
Fig. 7 Nelfinavir and bortezomib significantly reduce tumor volume in ELT3-V3 mouse xenografts, likely mediated through increased CHOP activity. ELT3-V3 tumor xenografts ( $n=14$ per group) were treated with either vehicle control, $5 \mathrm{mg} / \mathrm{ml}$ nelfinavir (NFV), $0.05 \mathrm{mg} / \mathrm{ml}$ bortezomib (BTZ), or NFV and BTZ in combination by intraperitoneal injection. a Tumor volume was recorded over 35 days before remaining mice were euthanized. Significant reduction of tumor volume was observed at day 35 when comparing combination to vehicle control. b Representative images of tumors stained with hematoxylin (to indicate cell nuclei, blue) and an antibody against $\mathrm{CHOP}$ (brown). The percentage of CHOP-positive cells per treatment is indicated. c Western blot for ATF4, PARP cleavage, or $\beta$-actin were carried out in triplicate per treatment
A

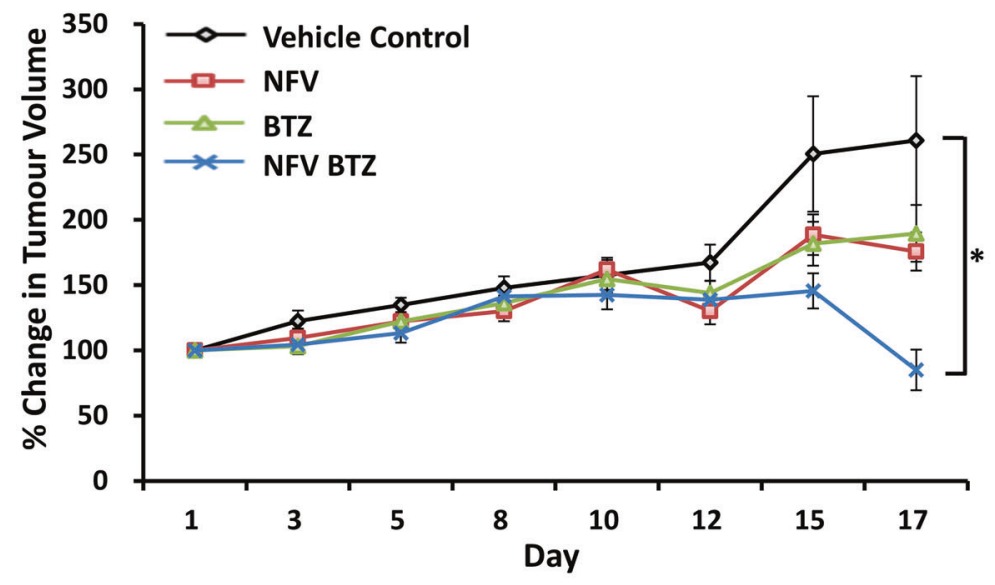

B

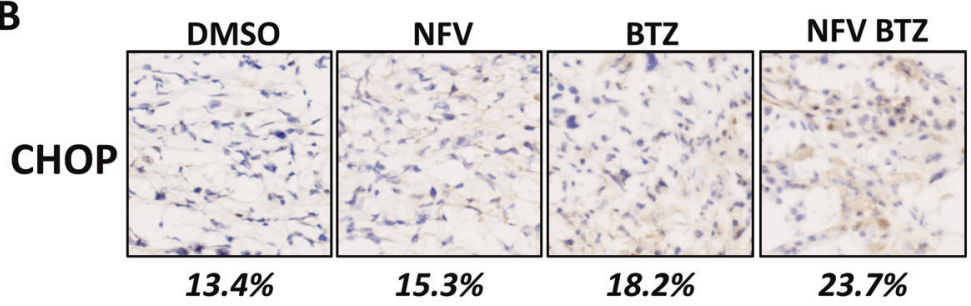

C

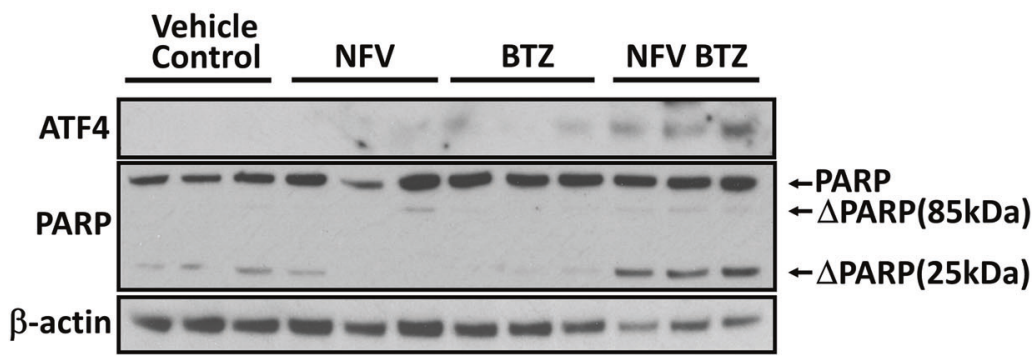

activity against renal tumors in $T s c 2+/-$ mice as a single agent [9], perhaps reflecting a failure to induce a sufficient level of ER stress. This problem could potentially be overcome by combining two ER stress inducing agents, such as nelfinavir and bortezomib.

Bortezomib (Velcade, Janssen-Cilag) was the first FDAapproved proteasome inhibitor that showed clinical promise for treating cancer. Bortezomib was approved for advanced multiple myeloma and more recently for mantle cell lymphoma. Next-generation proteasome inhibitors (marizomib and carfilzomib) are currently in clinical trials. Bortezomib's specifically binds the catalytic site of the $26 \mathrm{~S}$ proteasome to inhibit enzyme activity. By inhibiting the ubiquitin-proteasome system, bortezomib markedly alters the survival status of cancer cells. The synergy observed between nelfinavir and bortezomib is unlikely due to ER stress alone, but probably involves other processes. Additional processes affected upon proteasome inhibition include cell cycle control, apoptosis, angiogenesis, transcriptional regulation, and DNA-damage response (see review [21]). Although the nelfinavir and bortezomib combination showed considerable toxicity in mice in our study, a recent phase I clinical trial (clinicaltrials.gov: NCT01164709) in bortezomib-refractory multiple myeloma combining bortezomib with nelfinavir was well tolerated, safe and showed activity [11]. A phase II trial of advanced hematological malignancies showed that nine relapse patients whose malignancies were resistant to bortezomib had either a partial response or clinical benefit when bortezomib was combined with nelfinavir, with no apparent increase in toxicity [11].

Our work demonstrates for the first time that functional loss of TSC2 and subsequent mTORC1 hyperactivation sensitizes cells to combined proteasomal inhibition and ER stress. Our findings have clinical relevance in stratified medicine, where cancers with compromised signal transduction through TSC1/2-mTORC1 (via upstream pathways, e.g., oncogenic K-RAS or loss of PTEN) may be sensitive to nelfinavir and bortezomib. Our data imply that a high ER stress burden and hyperactive mTORC1 could function as 
predictive biomarkers of drug efficacy when considering combined nelfinavir and bortezomib treatment.

\section{Materials and methods}

\section{Cell culture and reagents}

Tsc2+/+ p53-I- and Tsc2-I- p53-I- MEFs were kindly provided by D. Kwiatkowski in 2004 (Harvard University, Boston, USA) [22]. Eker rat leiomyoma-derived Tsc2-deficient cells (ELT3-V3) and matched controls reexpressing Tsc2 (ELT3-T3) were generated by Astrinidis et al. [17] and were gifted in 2006 by C. Walker (M.D. Anderson Cancer Center, Houston, USA). HCT116 cells were provided by N. Leslie (Heriot Watt University, Edinburgh) in 2015, while human lung carcinoma (NCIH460) cells were bought from ATCC (in 2012). All cell lines were regularly screened for mycoplasma using the Venor GeM Classic PCR kit (CamBio) and were mycoplasma free. Cells were cultured in Dulbecco's modified Eagle's medium (BE12-604F, Lonza, Basel, Switzerland) supplemented with $10 \% \quad(\mathrm{v} / \mathrm{v})$ foetal bovine serum (10270106, Thermo Fisher Scientific), $100 \mathrm{U} / \mathrm{ml}$ penicillin and $100 \mu \mathrm{g} / \mathrm{ml}$ streptomycin (P4333, Sigma-Aldrich, Dorset, UK) at $37^{\circ} \mathrm{C}, 5 \%(\mathrm{v} / \mathrm{v}) \mathrm{CO}_{2}$. Nelfinavir mesylate hydrate (PZ0013), MG132 (C2211), thapsigargin (T9033), and etoposide (E1383) were purchased from Sigma. Bortezomib (CAS 179324-69-7), rapamycin (CAS 53123-889), Z-VAD-FMK (CAS 161401-82-7), and GSK2606414 were from Merck Millipore (Hertfordshire, UK).

\section{mRNA extraction, reverse transcription, XBP1 splicing, Chop, and Bip qPCR}

Samples were prepared and analyzed as described previously [15]. Bip was analyzed using Quantitect primers (QT00172361, Qiagen).

\section{Western blotting}

Cells were washed in PBS and lysed in radio immunoprecipitation assay (RIPA) buffer (R0278) supplemented with Complete Mini protease inhibitor cocktail (11836170001), PhosSTOP phosphatase inhibitor cocktail (04906837001) and $1 \mathrm{mM}$ dithiothreitol (DTT, D0632) (all from Sigma). Detached dead cells in the media and PBS wash were collected by centrifugation for $5 \mathrm{~min}$ at $5000 \mathrm{rpm}$ and combined with the lysate. After sonication, equal protein amounts were loaded on SDS-PAGE and western blotting was performed as described previously [23]. Protein from xenografts was extracted using the AllPrep DNA/RNA/Protein Mini Kit (Qiagen). Antibodies towards C/EBP homologous protein
(CHOP, \#2895), inositol-requiring and ER-to-nucleus signaling protein $1 \alpha$ (IRE1 $\alpha, \# 3294 S)$, ATF4 (\#11815), caspase 3 (\#9662), caspase 8 (mouse specific \#4927, human specific \#9746), PARP (\#9542), TSC2 (\#3990), and $\beta$-actin (\#4967) were purchased from Cell Signaling Technology (Danvers, USA). Growth arrest and DNA damage-inducible protein 34 (GADD34, also known as Protein phosphatase 1 regulatory subunit 15A [PPP1R15A], 10449-1-AP) antibodies were bought from Proteintech (Manchester, UK). Ubiquitin antibodies were from BioMol (PW8810). Densitometry was performed using ImageJ (version 1.51j8).

\section{Late cell death assay and determination of drug synergy}

Cell death assays were performed as previously described [15]. To determine synergy, a range of drug concentrations was used and the CI values were calculated using CompuSyn software (ComboSyn, Inc.) using a non-constant ratio approach.

\section{DNA fragmentation ELISA}

DNA fragmentation was determined with the Cell Death Detection ELISA kit (Roche). Immobilized histone complex was quantified photometrically (at $405 \mathrm{~nm}$ ) using 2,2,0-azino-bis-3-ethylbenzothiazoline-6-sulfonic acid as a peroxidase substrate.

\section{Proteasome activity analysis}

Proteasomes were extracted $2 \mathrm{~h}$ post-treatment and their chymotrypsin-like proteasome activity determined as described [24].

\section{Soft agar assay, spheroids, and outgrowth}

Soft agar assays, spheroid formation, and outgrowth analysis were performed as described [25]. For phalloidin staining, spheroids were fixed in $4 \%$ paraformaldehyde for $30 \mathrm{~min}$. Spheroids were permeabilized using $0.1 \%$ TritonX100 for 45 min before being stained with ActinGreen 488 Ready Probes Reagent (R37110, Thermo Fisher). Spheroids were stained with $3 \mu \mathrm{M}$ DRAQ7 (DR71000, Biostatus), transferred to a glass-bottomed plate, imaged using a Zeiss LSM 880 confocal microscope with Zen software and then analyzed using ImageJ v1.50i.

\section{RNA-Seq sample preparation, sequencing, and analysis}

RNA quality was assessed using Agilent 2100 Bioanalyser and an RNA Nano 6000 kit (Agilent Technologies). 
100-900 ng of total RNA with an RIN value $>8$ was depleted of ribosomal RNA. Sequencing libraries were prepared using the Illumina ${ }^{\oplus}$ TruSeq ${ }^{\oplus}$ Stranded total RNA with Ribo-Zero Gold ${ }^{\mathrm{TM}}$ kit (Illumina Inc.). Steps include: rRNA depletion/cleanup, RNA fragmentation, 1st strand cDNA synthesis, 2nd strand cDNA synthesis, adenylation of 3'-ends, adapter ligation, PCR amplification (12-cycles) and validation. The manufacturer's protocol was followed except for the cleanup after the ribozero depletion step where Ampure XP beads (Beckman Coulter) and $80 \%$ ethanol were used. Libraries were validated using the Agilent 2100 Bioanalyser with a high-sensitivity kit (Agilent Technologies) to determine insert size, and quantified with Qubit $^{\circledast}$ (Life Technologies). Libraries were normalized to $4 \mathrm{nM}$, pooled and clustered on $\operatorname{cBot}^{\mathrm{TM}} 2$ following the manufacturer's protocol. Sequencing used a 75-base pairedend $\left(2 \times 75\right.$ bp PE) dual index read format on the Illumina ${ }^{\oplus}$ HiSeq2500 (high-output mode). Quality control checks were performed using FastQC before mapping to the UCSC mouse mm10 reference genome (using Tophat and Bowtie). Differentially expressed transcripts were identified using DeSeq2 analysis [26] on normalized count data with the design formula setup to analyze all pairwise comparisons in the dataset using contrasts. $P$ values were corrected with the FDR method. Genes based on GO:0008219 (cell death) were selected from the complete list on AmiGo 2 (http://a migo.geneontology.org/amigo/landing).

\section{Protein translation assay}

Performed as in ref. [27], using EasyTag ${ }^{\mathrm{TM}}$ EXPRESS- $\left[{ }^{35} \mathrm{~S}\right]$ Protein Labeling Mix (NEG772007MC, Perkin Elmer).

\section{ELT-3 mouse xenograft}

Animal experimental procedures were approved by the Institutional Animal Care and Use Committee of CrownBIO. Care and use of animals was conducted in accordance with the regulations of the Association for Assessment and Accreditation of Laboratory Animal Care (AAALAC). A mouse xenograft model was established using ELT3-V3 cells inoculated into 9-10-week-old female NOD/SCID mice (HFK Bio-Technology Co. Ltd. (Beijing, China)). Sample size was based upon using a two-tailed $t$ test, assuming unequal variance and large effect size of 0.8 with $60 \%$ power at the $10 \%$ significance level. Exponentially growing ELT3V3 cells were used for tumor inoculation. One week before cell inoculation, mice were implanted with 17- $\beta$-estradiol pellets $(2.5 \mathrm{mg}$, 90-day release, Innovative Research of America), then inoculated subcutaneously at the right flank with ELT3-V3 cells $\left(5 \times 10^{6}\right)$ in $0.2 \mathrm{ml}$ of PBS. Tumor volumes were measured in two dimensions using a calliper (volume $\left(\mathrm{mm}^{3}\right)=0.5 a \times b^{2}$; where a and $b$ are the long and short diameters of the tumor, respectively). Grouping and treatments began after the mean tumor size reached $186 \mathrm{~mm}^{3}$. Fourteen mice were assigned per treatment group using a randomized block design, based on their tumor volumes to receive either: (1) vehicle (4\% (v/v) DMSO, 5\% (v/v) PEG, $5 \%(\mathrm{v} / \mathrm{v})$ TWEEN 80 in saline); (2) nelfinavir, $50 \mathrm{mg} / \mathrm{kg}$ dissolved in vehicle; (3) bortezomib, $0.5 \mathrm{mg} / \mathrm{kg}$ dissolved in $0.04 \%$ (v/v) mannitol solution; or (4) nelfinavir, $50 \mathrm{mg} / \mathrm{kg}$ and bortezomib, $0.5 \mathrm{mg} / \mathrm{kg}$. Treatments were administered intraperitoneally on days $1,3,5,8,10,12,15$ and 17 . Dosages were reduced to $30 \mathrm{mg} / \mathrm{kg}$ nelfinavir and $0.3 \mathrm{mg} / \mathrm{kg}$ bortezomib on day 8 due to toxicity. Tumor volumes were measured three times per week. Investigators were not blinded to the group allocation. Due to lower numbers of mice than anticipated at day 17, groups were compared nonparametrically using the Kruskal-Wallis test and pairwise comparisons.

\section{Immunohistochemistry}

Tumors in optimal cutting temperature compound were snap frozen and cryostat sectioned at $10 \mu \mathrm{m}$ thickness. Sections were warmed to room temperature for $30 \mathrm{~min}$, fixed in ice-cold acetone (5 $\mathrm{min})$ and air-dried $(30 \mathrm{~min})$. After blocking in 5\% (v/v) normal goat serum in TrisBuffered Saline (pH 7.6) 0.1\% (v/v) Tween-20, sections were incubated at $4{ }^{\circ} \mathrm{C}$ with $1 / 1000$ rabbit monoclonal antibody against CHOP (Abcam, ab179823) overnight, blocked with Envision peroxidase block and incubated for $30 \mathrm{~min}$ in Envision rabbit polymer, before detection with DAB chromogen (all DAKO). Slides were hematoxylin counterstained, dehydrated through an ethanol series and xylene, before mounting in DPX medium (Fisher Scientific). Five fields from each tumor were scored for $\%$ of CHOP-positive cells (ImageJ, v1.51j8).

\section{Statistical analysis}

At least three independent, biological repeats were performed for each experiment. Exact sample size is indicated in each figure legend. Results are expressed as mean \pm standard deviation (SD), unless otherwise specified in the figure legend. Data analysis was carried out using a oneway ANOVA followed by LSD post-hoc test, or an independent samples Kruskal-Wallis test as appropriate. Significance is reported at $* p<0.05, * * p<0.01, * * * p<0.001$, and NS $=$ not significant.

Acknowledgements This work was supported by Health and Care Research Wales (the Wales Cancer Research Centre) (to EAD, ART, PJG and TH), the Tuberous Sclerosis Association (to EAD, ART, and MHS), Cancer Research Wales (to CEJ, RJE, and ART), the Tuberous Sclerosis Alliance (to EAD and ART), the Hospital Saturday Fund (to ART) and BBSRC (to RJE). We would like to thank the Wales Gene Park for their contribution to this study. 


\section{Compliance with ethical standards}

Conflict of interest RJE is non-executive director of Biostatus Ltd, the vendor of DRAQ7. The remaining authors declare that they have no conflict of interests.

Open Access This article is licensed under a Creative Commons Attribution 4.0 International License, which permits use, sharing, adaptation, distribution and reproduction in any medium or format, as long as you give appropriate credit to the original author(s) and the source, provide a link to the Creative Commons license, and indicate if changes were made. The images or other third party material in this article are included in the article's Creative Commons license, unless indicated otherwise in a credit line to the material. If material is not included in the article's Creative Commons license and your intended use is not permitted by statutory regulation or exceeds the permitted use, you will need to obtain permission directly from the copyright holder. To view a copy of this license, visit http://creativecommons. org/licenses/by/4.0/.

\section{References}

1. Clarke HJ, Chambers JE, Liniker E, Marciniak SJ. Endoplasmic reticulum stress in malignancy. Cancer Cell. 2014;25:563-73.

2. Appenzeller-Herzog C, Hall MN. Bidirectional crosstalk between endoplasmic reticulum stress and mTOR signaling. Trends Cell Biol. 2012;22:274-82.

3. Dunlop EA, Tee AR. mTOR and autophagy: a dynamic relationship governed by nutrients and energy. Semin Cell Dev Biol. 2014;36:121-9.

4. Stengel S, Messner B, Falk-Paulsen M, Sommer N, Rosenstiel P. Regulated proteolysis as an element of ER stress and autophagy: implications for intestinal inflammation. Biochim Biophys Acta. 2017;1864:2183-90.

5. Lam HC, Nijmeh J, Henske EP. New developments in the genetics and pathogenesis of tumours in tuberous sclerosis complex. J Pathol. 2017;241:219-25.

6. Zhang Y, Nicholatos J, Dreier JR, Ricoult SJ, Widenmaier SB, Hotamisligil GS, et al. Coordinated regulation of protein synthesis and degradation by mTORC1. Nature. 2014;513:440-3.

7. Siroky BJ, Yin H, Babcock JT, Lu L, Hellmann AR, Dixon BP, et al. Human TSC-associated renal angiomyolipoma cells are hypersensitive to ER stress. Am J Physiol Ren Physiol. 2012;303: F831-844.

8. Babcock JT, Nguyen HB, He Y, Hendricks JW, Wek RC, Quilliam LA. Mammalian target of rapamycin complex 1 (mTORC1) enhances bortezomib-induced death in tuberous sclerosis complex (TSC)-null cells by a c-MYC-dependent induction of the unfolded protein response. J Biol Chem. 2013;288:15687-98.

9. Auricchio N, Malinowska I, Shaw R, Manning BD, Kwiatkowski DJ. Therapeutic trial of metformin and bortezomib in a mouse model of tuberous sclerosis complex (TSC). PLoS ONE 2012;7: e31900.

10. Gills JJ, Lopiccolo J, Tsurutani J, Shoemaker RH, Best CJ, AbuAsab MS, et al. Nelfinavir, A lead HIV protease inhibitor, is a broad-spectrum, anticancer agent that induces endoplasmic reticulum stress, autophagy, and apoptosis in vitro and in vivo. Clin Cancer Res. 2007;13:5183-94.

11. Driessen C, Kraus M, Joerger M, Rosing H, Bader J, Hitz F, et al. Treatment with the HIV protease inhibitor nelfinavir triggers the unfolded protein response and may overcome proteasome inhibitor resistance of multiple myeloma in combination with bortezomib: a phase I trial (SAKK 65/08). Haematologica. 2016;101:346-55.
12. Shim JS, Rao R, Beebe K, Neckers L, Han I, Nahta R, et al. Selective inhibition of HER2-positive breast cancer cells by the HIV protease inhibitor nelfinavir. $J$ Natl Cancer Inst. 2012;104:1576-90.

13. Kraus M, Müller-Ide H, Rückrich T, Bader J, Overkleeft H, Driessen C. Ritonavir, nelfinavir, saquinavir and lopinavir induce proteotoxic stress in acute myeloid leukemia cells and sensitize them for proteasome inhibitor treatment at low micromolar drug concentrations. Leuk Res. 2014;38:383-92.

14. Kawabata S, Gills JJ, Mercado-Matos JR, Lopiccolo J, Wilson W 3rd, Hollander MC, et al. Synergistic effects of nelfinavir and bortezomib on proteotoxic death of NSCLC and multiple myeloma cells. Cell Death Dis. 2012;3:e353.

15. Johnson CE, Hunt DK, Wiltshire M, Herbert TP, Sampson JR, Errington RJ, et al. Endoplasmic reticulum stress and cell death in mTORC1-overactive cells is induced by nelfinavir and enhanced by chloroquine. Mol Oncol. 2015;9:675-88.

16. Kraus M, Bader J, Overkleeft H, Driessen C. Nelfinavir augments proteasome inhibition by bortezomib in myeloma cells and overcomes bortezomib and carfilzomib resistance. Blood Cancer J. 2013;3:e103.

17. Astrinidis A, Cash TP, Hunter DS, Walker CL, Chernoff J, Henske EP. Tuberin, the tuberous sclerosis complex 2 tumor suppressor gene product, regulates Rho activation, cell adhesion and migration. Oncogene. 2002;21:8470-6.

18. Peng ZF, Yang L, Wang TT, Han P, Liu ZH, Wei Q. Efficacy and safety of sirolimus for renal angiomyolipoma in patients with tuberous sclerosis complex or sporadic lymphangioleiomyomatosis: a systematic review. J Urol. 2014;192:1424-30.

19. Dabora SL, Franz DN, Ashwal S, Sagalowsky A, DiMario FJ Jr, Miles D, et al. Multicenter phase 2 trial of sirolimus for tuberous sclerosis: kidney angiomyolipomas and other tumors regress and VEGF- D levels decrease. PLoS ONE 2011;6:e23379.

20. Kang YJ, Lu MK, Guan KL. The TSC1 and TSC2 tumor suppressors are required for proper ER stress response and protect cells from ER stress-induced apoptosis. Cell Death Differ. 2011;18:133-44.

21. Gandolfi S, Laubach JP, Hideshima T, Chauhan D, Anderson KC, Richardson PG. The proteasome and proteasome inhibitors in multiple myeloma. Cancer Metastas- Rev. 2017;36:561-84.

22. Zhang H, Cicchetti G, Onda H, Koon HB, Asrican K, Bajraszewski N, Vazquez F, Carpenter CL, Kwiatkowski DJ. Loss of Tsc1/Tsc2 activates mTOR and disrupts PI3K-Akt signaling through downregulation of PDGFR. $\mathrm{J}$ Clin Invest. 2003;112:1223-33.

23. Dunlop EA, Hunt DK, Acosta-Jaquez HA, Fingar DC, Tee AR. ULK1 inhibits mTORC1 signaling, promotes multisite Raptor phosphorylation and hinders substrate binding. Autophagy. 2011;7:737-47.

24. Crawford LJ, Walker B, Ovaa H, Chauhan D, Anderson KC, Morris TC, et al. Comparative selectivity and specificity of the proteasome inhibitors BzLLLCOCHO, PS-341, and MG-132. Cancer Res. 2006;66:6379-86.

25. Dunlop EA, Johnson CE, Wiltshire M, Errington RJ, Tee AR. Targeting protein homeostasis with nelfinavir/salinomycin dual therapy effectively induces death of mTORC1 hyperactive cells. Oncotarget. 2017;8:48711-24.

26. Love MI, Huber W, Anders S. Moderated estimation of fold change and dispersion for RNA-seq data with DESeq2. Genome Biol. 2014;15:550.

27. Tee AR, Proud CG. DNA-damaging agents cause inactivation of translational regulators linked to mTOR signalling. Oncogene. 2000;19:3021-31. 